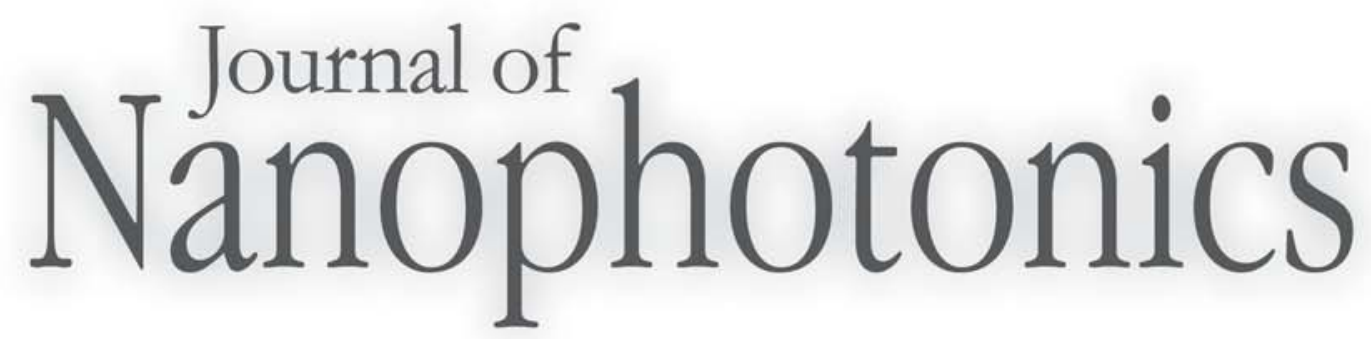

SPIEDigitallibrary.org/jnp

\title{
Commentary: Multiple surface- plasmon-polariton waves at a single bi-material interface
}

John A. Polo, Jr. 


\title{
Commentary: Multiple surface-plasmon-polariton waves at a single bi-material interface
}

\author{
John A. Polo, Jr. \\ Edinboro University of Pennsylvania, Department of Physics and Technology, \\ 235 Scotland Road, Edinboro, PA 16444, USA \\ polo@edinboro.edu
}

Electromagnetic surface waves have been a subject of investigation since the beginning of the $20^{\text {th }}$ century when Zenneck proposed that radio waves might propagate as surface waves bound to the air/Earth interface. Although that possibility is still being debated, the midtwentieth century saw the resurfacing of this idea with application to waves at optical frequencies bound to metal/insulator interfaces. In this regime, the surface wave is known as a surface-plasmon-polariton (SPP) wave. Within the past few decades, SPP waves have proved to be extremely valuable as a means of creating sensitive chemical sensors, especially for the detection of biochemicals [1,2]. As the ability to manipulate materials at the microscopic scale has developed, improved implementation of SPP waves with various structures and geometries continue to be proposed and realized. It is well known that the advent of nanotechnology has brought about composite materials with properties that can be quite different than those of the constituent materials in the bulk. Recent investigations [3-8] have shown that interfaces of nanostructured periodic dielectrics and metals can support SPP waves in a very novel way.

SPP waves travel, bound to a metal/dielectric material interface, with an amplitude that decreases with distance from the interface. When the dielectric material is a simple homogeneous, isotropic material, the amplitude decreases exponentially with distance from the interface, with a more rapid decrease on the metal side of the interface than the dielectric side. The fields drop to insignificant values at distances on the order of $100 \mathrm{~nm}$ from the interface on the dielectric side. It is the tight confinement of the fields to the interface that is responsible for the extreme sensitivity of SPP-wave chemical sensors. Only a small number of molecules in the very narrow region near the interface results in a significant modification of the the SPP wave.

An SPP wave can not be excited directly by shining light at the interface, because of a mismatch between the wavevector components parallel to the surface of the interface of light beam and the SPP wave. Various methods are used to couple an exciting beam of light to the SPP wave. The most popular method of excitation is accomplished with a coupling prism on the metal side of the interface. This arrangement, known as the Kretschmann configuration, is shown schematically in Fig. 1. In this configuration, the metal forming the interface is first deposited as a very thin film on the prism before being placed in contact with the partnering dielectric material, often a liquid. Under the right conditions, the exciting beam couples to the SPP wave. At this resonance, energy of the exciting beam is absorbed by the SPP wave and is observed as a decrease in reflectance form the interface. The SPP wave is commonly observed be either varying the angle of incidence of a fixed wavelength of exciting light, or varying wavelength of exciting light at a fixed angle of incidence in order to bring the exciting beam into resonance with the SPP wave. In either case, a sharp dip in the reflectance is observed when the SPP wave is excited and energy is absorbed from the exciting wave. The angular or spectral position of the dip in reflectance is highly dependant on the index of refraction of the dielectric material. A shift in the position of the peak, upon exposure of the interface to the environment under analysis, signals the detection of an analyte.

Sculptured thin films (STFs) are a class of nanostructured materials formed by directing a columnated beam of chemical vapor at a substrate in a vacuum. Under appropriate conditions, the deposited material self-assembles into parallel columns with diameters on the order of 20 


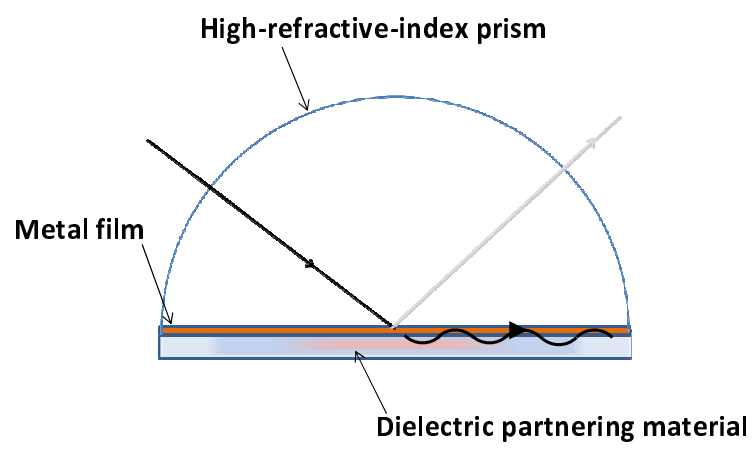

Fig. 1. Kretschmann configuration

to $100 \mathrm{~nm}$. By rotating and/or rocking the substrate during deposition, the columns can be sculptured into various shapes. Rocking the substrate results in a sculptured nematic thin film (SNTF) with columns having a planar morphology; while, slowly rotating the substrate during deposition results in a chiral STF which consists of helical coils with axes oriented perpendicularly to the surface of the substrate. STFs have a number of interesting mechanical and optical properties which have been the subject of theoretical and experimental investigations for several decades. Among the proposed optical applications of STF are: optical filters, mirrors and chemical sensors. During the past three years, several investigations have pursued a new use of STFs as the partnering dielectric material forming the interface for SPP-wave propagation.

Investigation started with simpler columnar thin films (CTFs) [9]. CTFs are formed by directing the columnated beam of vapor at a stationary substrate, resulting in straight columns projecting, in general, at an angle to the surface. As the size of the columns is appreciably smaller than optical wavelengths, the CTF appears homogeneous to light at optical wavelengths. The material is, however, anisotropic, as the columns have, in general, a non-circular crosssection. As might be expected, the characterization of the SPP wave with a CTF instead of an isotropic dielectric material is more complicated. The properties of a SPP wave bound to a metal/CTF interface, such as phase velocity and the degree of localization to the interface, depend on the orientation of the plane of incidence relative to the CTF columns.

Truly surprising results were revealed when calculations [4] were directed at SPP waves bound to the interface of a metal and the more complicated chiral STF. Multiple SPP-wave modes of propagation bound to a single interface for a single frequency of excitation were found. Although multiple modes are common for waveguide structures with more than one bounding surface, multiple modes had not been observed previously for SPP waves. The number of modes of SPP-wave propagation observed depended on the periodicity of the chiral STF, with as many as four modes for a single metal/chiral STF interface. Later calculations $[3,5]$ would reveal the same phenomenon at the interface of a metal and a periodic SNTF. In most cases, SPP waves could be observed, with an STF comprised of only two structural cycles, and, furthermore, the SPP waves were nearly identical to SPP waves calculated at the interface of semi-infinite expanses of metal and STF with an infinite number of cycles. For a few SPP-wave modes at particular periodicities, larger number of STF cycles were required, but they still numbered only twenty or thirty. This is an important finding since STFs fabricated with a large number of cycles begin to lose regularity. The existence of multiple SPP waves have since been confirmed experimentally for both metal/periodic SNTF interfaces [6] and metal/chiral STF interfaces [7].

The field profiles [8] of the SPP wave at the metal/STF interface show a richness not observed at metal/isotropic dielectric material interfaces. Unlike the simple exponential decay of amplitude with distance from the interface observed for SPP waves bound to metal/isotropic dielectric material interfaces, SPP waves bound to the metal/STF interface show much more complex decay patterns, on the STF side of the interface, which vary from one mode to another. 
In addition to the variability in the shape of the profile, the distance over which the fields decay to inappreciable levels varies significantly among the modes.

Calculations showed that incident light with polarization either parallel to the plane of incidence ( $p$ polarization) or perpendicular to the plane of incidence ( $s$ polarization) could be used to excite some SPP-wave modes. This contrasts to the behavior of SPP waves bound to metal/isotropic dielectric material interfaces, which may only be excited by $p$-polarized light. The excitation of SPP waves with s-polarized light, in the case of chiral STF dielectric, may not be too surprising, since it might be argued that some conversion of $s$-polarization to $p$-polarization could occur because of the helical nature of the nanocolumns. This mechanism, however, can not be invoked to explain the results of calculations [3] which show that $s$-polarized light traveling parallel to the morphologically significant plane of the SNTF, at a metal/SNTF interface, can also excite a SPP wave.

The use of periodic STFs to form metal/dielectric material interfaces for the support of SPP waves offers at least two exciting areas for investigation: exploitation of the complex porous environment of the STF, and the use of multiple SPP-wave modes. The porosity of the STF offers a path for molecular species to come into close contact with the interface, yet may filter out larger particulate matter in a dirty environment. The porosity and large surface area may also provide a supportive environment for holding recognition molecules commonly used to bind biomolecular analytes to the surface for detection. With recognition molecules embedded in the protective environment of the STF, SPP-wave detectors might become more suitable for use in the field. Of course, the existence of multiple SPP-wave modes bound to a single interface is the most unique feature of the metal/STF interface. Multiple SPP-wave modes may provide a means of error free chemical sensing with a single metal/dielectric material interface.

Perhaps the most exciting possibility, the ability to detect more than one species with a single interface, is a result of both the porous structure of the STF and its ability to support multiple SPP-wave modes. As the STF provides a complex structural environment, different analyte molecules will undoubtedly diffuse and orient themselves differently in the porous structure of the STF. With the multiple SPP-wave modes characterized by various field profiles and polarizations, each mode is likely to have a characteristic response to various analytes.

Metal/STF interfaces supporting multiple SPP-wave modes offer a rich palette of parameters with which to design detectors: a wide variety of materials for the construction of the STF, a limitless variety of STF conformations and periodicities, post processing of the STF to modify properties of the film, and two polarizations for excitation. It should also be mentioned that, although calculations to date were performed on metal/STF interfaces, an interface of a metal and other periodic dielectric materials could also support multiple SPP-wave modes. The use of liquid crystals [10], for instance, might offer dynamic, voltage controlled, SPP-wave detectors. Determining how to implement multiple SPP modes in a new generation of SPP-wave detectors is sure to provide an exciting avenue of research for several years to come.

\section{References}

1. J. Homola (ed.), Surface Plasmon Resonance Based Sensors, Springer, Heidelberg, Germany (2006) [doi:10.1007/b100321].

2. I. Abdulhalim, M. Zourob, and A. Lakhtakia, "Surface plasmon resonance for biosensing: A mini-review," Electromagnetics 28, 214-242 (2008) [doi:10.1080/02726340801921650].

3. M.A. Motyka and A. Lakhtakia, "Multiple trains of same-color surface plasmonpolaritons guided by the planar interface of a metal and a sculptured nematic thin film," $J$. Nanophoton. 2, 021910 (2008) [doi:10.1117/1.3033757].

4. J.A. Polo Jr and A. Lakhtakia, "On the surface plasmon polariton wave at the planar interface of a metal and a chiral sculptured thin film," Proc. R. Soc. Lond. A 465, 87-107 (2009) [doi:10.1098/rspa.2008.0211]. 
5. M.A. Motyka and A. Lakhtakia, "Multiple trains of same-color surface plasmonpolaritons guided by the planar interface of a metal and a sculptured nematic thin film. Part II: Arbitrary incidence,” J. Nanophoton. 3, 033502 (2009) [doi:10.1117/1.3147876].

6. A. Lakhtakia, Y.-J. Jen, and C.-F. Lin, "Multiple trains of same-color surface plasmonpolaritons guided by the planar interface of a metal and a sculptured nematic thin film. Part III: Experimental evidence," J. Nanophoton. 3, 033506 (2009) [doi:10.1117/1.3249629].

7. Devender, D.P. Pulsifer, and A. Lakhtakia, "Multiple surface plasmon polariton waves," Electron. Lett. 45, 1137-1138 (2009) [doi:10.1049/el.2009.2049].

8. J.A. Polo Jr. and A. Lakhtakia, "Energy flux in a surface-plasmon-polariton wave bound to the planar interface of a metal and a structurally chiral material," J. Opt. Soc. Am. A 26, 1696-1703 (2009) [doi:10.1364/JOSAA.26.001696].

9. J.A. Polo Jr., S.R. Nelatury, and A. Lakhtakia, "Propagation of surface waves at the planar interface of a columnar thin film and an isotropic substrate," J. Nanophoton. 1, 013501 (2007) [doi:10.1117/1.2515618].

10. P.G. de Gennes and J. Prost, The Physics of Liquid Crystals, 2nd ed., Clarendon Press, Oxford, United Kingdom (1993). 